(C) (C) 2019 IEEE. Personal use of this material is permitted. Permission from IEEE must be obtained for all other uses, in any current or future media, including reprinting/republishing this material for advertising or promotional purposes, creating new collective works, for resale or redistribution to servers or lists, or reuse of any copyrighted component of this work in other works 


\title{
Multi-DoF Force Characterization of Soft Actuators
}

\author{
Sagar Joshi and Jamie Paik
}

\begin{abstract}
Soft actuators provide highly adaptable actuator options for applications in wearable devices, grippers and mobile robots due to their inherent compliance. However, this compliance causes soft actuators to have virtually infinite degrees of freedom (DoF) of motion, and challenges accurate prediction of their displacement and interaction forces. While several studies have characterized soft actuators either in blocked condition or in a specific range of motion, the testing conditions often do not match actual loading conditions, which leads to discrepancies between expected and observed mechanical behaviour.

Here, we propose a novel multi-DoF experimental protocol for characterizing soft actuator interaction forces by considering three critical aspects: anchoring conditions, displacement boundary conditions and actuation power. In order to conduct this multi-DoF characterization, we designed a novel reconfigurable robotic test platform for enforcing anchoring conditions and planar displacement boundary conditions, and measuring forces at multiple contact locations. Using this experimental protocol and set-up, we dictated three loading conditions: pulling force, tip loading and three-point bending; and analyzed the interaction forces of a soft bending actuator. Our results show that the three loading conditions produce distinct actuator behaviours. This validates the importance of loading conditions on soft actuator performance.
\end{abstract}

Index Terms-Soft Sensors and Actuators; Soft Robot Applications

\section{INTRODUCTION}

S OFT actuators present unique solutions in robotics because of characteristics such as compliance, conformability and versatility $[1]-[3]$. They are being used increasingly in applications including wearable devices [2], [4][10], locomotion [11]-[16] and grasping [17] $-[19]$. The overall performance of these actuators is defined by their mechanical behaviour while interacting with their environments. For instance, actuator displacement along with magnitude and direction of interaction forces governs the grasping ability for a gripper [18] or the step length for a walking robot [11]. Similarly for a wearable robot, the distribution of forces at the contact locations impacts overall effectiveness. In order to further the scope of soft robotics, an understanding of soft actuator mechanical behaviour is necessary.

There are numerous studies investigating soft actuator displacement and interaction forces using analytical [15], [20][26], numerical [2], [27]-[29] or experimental methods [4][7], [14], [15], [30]-[33]. One study uses a quasi-static analytical model to predict tip force interactions of fibre reinforced soft pneumatic actuators (SPAs) in bending [34]. Trivedi et al. have used Cosserat rod theory to model the

Manuscript received: February, 24, 2019; Revised June, 5, 2019; Accepted June, 21, 2019. This paper was recommended for publication by Editor KyuJin Cho upon evaluation of the Associate Editor and Reviewers' comments. This work was supported by the Swiss National Science Foundation (SNSF) "START" Project 513956. (Corresponding author: Jamie Paik.)

S. Joshi and J. Paik are with the Reconfigurable Robotics Lab (RRL), Swiss Federal Institute of Technology Lausanne, Lausanne 1015, Switzerland (email: sagar.joshi@epfl.ch; jamie.paik@epfl.ch)

Digital Object Identifier (DOI): see top of this page. planar force and displacement of a 9 degree of freedom (DoF) soft pneumatic manipulator [22]. Another recent study uses equivalent rigid-body dynamics to model dynamic motion and force interactions of a SPA [26]. While these models are able to predict the displacement and interaction forces, they are limited to the specific actuators with simple conditions such as tip loading or assumptions such as constant curvatures. It is difficult to apply these models for non-standard actuator designs and loading conditions. A different approach to studying and predicting interaction forces uses finite elemental method (FEM). An open source physics simulator, Simulation Open Framework Architecture (SOFA), uses FEM for realtime modelling and control of soft deformable robots [27], [28]. Another tool, the SPA-tool kit, uses FEM to optimize SPA design parameters for meeting specifications of desired force and displacement [29]. Many other studies use FEM to predict free displacement and force output of soft actuators [2], [34]. While such numerical methods can address a larger range of actuator designs and loading conditions, in practice, aspects such as friction, hysteresis, manufacturing inconsistencies and boundary conditions uncertainty affect the accuracy and applicability of these methods. Therefore, several studies resort to experimental characterization as it is a direct measurement of the interaction forces [4]-[7], [14], [15], [30]-[33]. Moreover, experimental testing is necessary for validating results from analytical or numerical methods.

Several studies characterizing soft actuators measure only the blocking force, by constraining actuator movement [6], [9], [35]. While this gives the peak force capability, it gives no information about how the force evolves with actuator displacement. In some other studies, interaction forces are measured as the actuator moves through a specific range of motion [30], [32], [36]. However, the enforced displacement, parametrized by a single variable such as distance or curvature, forms only a small subset of possible displacement as soft actuators have virtually infinite degrees of freedom. Furthermore, these studies do not consider how the actuator is constrained to its surroundings, which influences its displacement as well as interaction forces. For example, the nature of contact between the actuator and its surroundings for an inchworm robot [16], [37], [38] is significantly different than that in a gripper [17], [18] or an assistive glove [7], [9], even though the same actuator can potentially be used for all three applications. As a result, existing methods for characterization are not able to replicate true loading conditions of the target application, and the characterization results do not represent the expected actuator behaviour. Additionally, these studies are unable to convey information about the magnitude and direction of the resultant force, or about force distribution; as the force is measured at a single location, and in a single axis.

Here, we introduce a novel multi-DoF experimental protocol for characterizing soft actuator mechanical performance. 
This protocol addresses three critical factors that govern soft actuator behaviour: anchoring conditions, displacement boundary conditions and actuation power. In order to conduct such multi-DoF characterization, we designed, prototyped and tested a novel robotic platform. This modular and reconfigurable platform is capable of extensively characterizing soft actuators in diverse loading conditions, irrespective of soft actuator design and materials. Using this platform, we characterized a bending soft actuator in three loading conditions, and found independent and distinct soft actuator behaviour in each. By having a physical experimental set-up that can recreate loading conditions, we obtain accurate and repeatable results beyond just "blocked forces" that are often measured by several studies [6], [9], [35], thereby leading to an improved understanding of soft actuator mechanical behaviour. The main contributions of this work are:

- A novel multi-DoF experimental protocol for examining and characterizing soft actuators, based on physically simulating anchoring conditions, displacement boundary conditions, and actuation power

- Design of a novel robotic platform for planar characterization of extending, contracting and bending soft actuators

- Validation of effectiveness of the experimental protocol and the platform by testing a bending soft actuator in three loading conditions, that resulted in producing three independent and distinct behaviours.

\section{INTERACTION FORCES OF SOFT ACTUATORS}

In addition to actuator design properties, i.e. geometrical shape and material properties, the following aspects critically affect soft actuator displacement and interaction forces:

- Anchoring conditions: These correspond to the location and nature of contact between the soft actuator and its surroundings, representing the physical interface between the two.
- Displacement boundary conditions: These correspond to the actuator displacement at the contact locations.

- Actuation power: These represent the input to the actuator. It corresponds to air pressure for pneumatically powered robots, voltage for electrically powered robots, and so on.

In order to recreate true loading conditions, we physically simulate the above and measure interaction forces, such that the characterization data will reflect the expected actuator behaviour accurately. However, due to the large set of possible loading scenarios and virtually infinite degrees of freedom of soft actuators, it is evident that an exhaustive study with such an approach is vast. In this letter, we consider hinge and cantilever constraints as anchoring conditions, enforce planar displacement at contact locations, and demonstrate our experimental protocol with a bending actuator [39] in the following three loading conditions, which are frequently observed in literature [7], [9], [11], [12], [16]-[19], [37], [38]:

- Pulling force using double hinged constraints: Both ends of the actuator are constrained to hinge joints that allow free rotation, but prevent translational motion (Fig. 2A). An example of such a condition is observed in inchworm robots [16], [37], [38], or for producing motion to pull an object towards itself.

- Tip loading using cantilever-hinge constraints: One end of the actuator is fixed while the other end has a hinge constraint (Fig. 4A). Such a condition is observed in grippers [17]-[19] or in the legs of a walking robot [11], [12], in which one end is fixed to the robot body while the other is in contact with the ground.

- Three-point bending using hinge-cantilever-hinge constraint: The two ends of the actuator are constrained to hinge joints, and the middle section is fixed (Fig. 6A). Such a condition is seen in wearable robots, which typically have multiple contact locations. For instance, a glove in which one end is strapped to the fingertip, the other end to the back of the hand, and the middle section is strapped to the knuckles [7], [9].
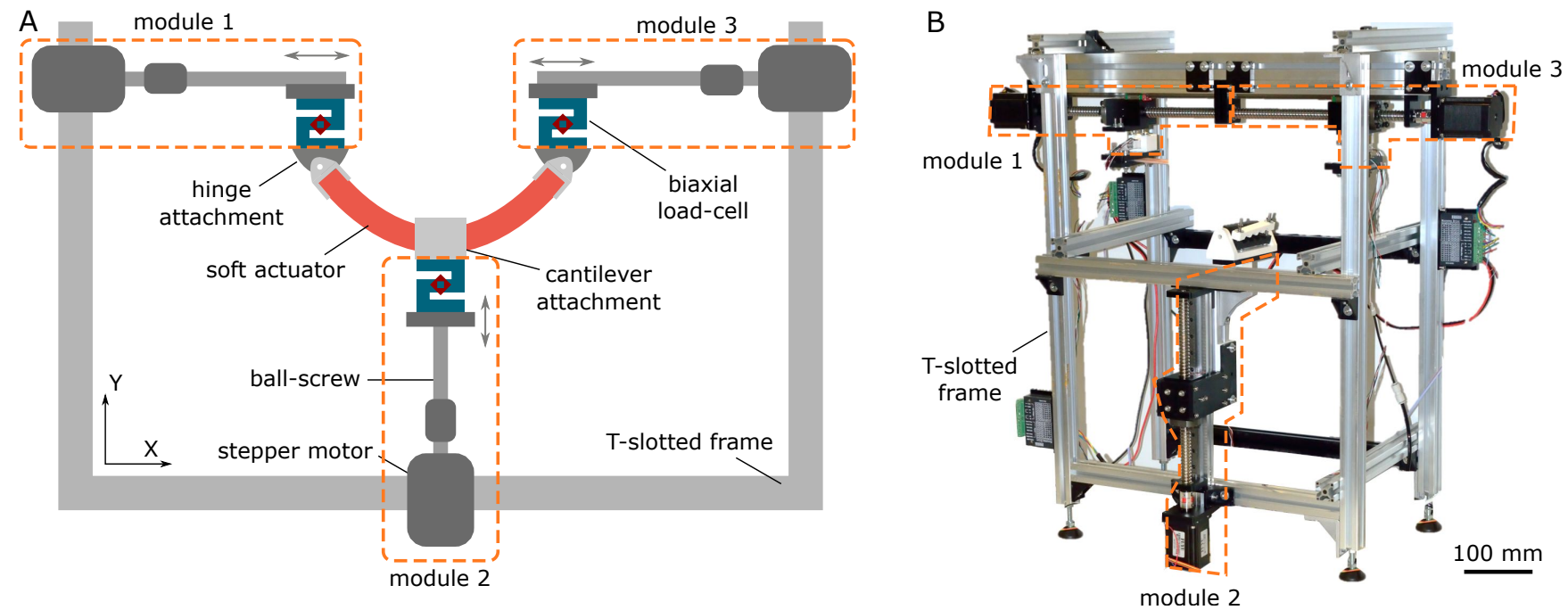

Fig. 1. Experimental setup: A novel robotic platform for characterization of soft actuators. The reconfigurable device consists of a rigid frame for structural support, attachments for enforcing anchoring conditions, and modules with motors and biaxial load-cells for enforcing displacement boundary conditions and for measuring interaction forces respectively. (A) Schematic showing the different components, and (B) The fabricated prototype. 


\section{EXPERIMENTAL SETUP AND PROTOCOL}

Here we characterize a bending soft actuator [39] in the three loading conditions defined previously: pulling force, tip loading and three-point bending.

\section{A. Experimental setup}

We designed a novel robotic platform (Fig. 1) for conducting multi-DoF characterization of soft actuators. By virtue of its modular and reconfigurable design, this device can be used to characterize soft actuators of different sizes and actuation mechanisms, namely extension, contraction and bending. It has three main components:

- Modules: These are used for enforcing displacement boundary conditions, and for measuring interaction forces at the contact locations. Each module has a linear motor (Fuyu Motion) consisting of stepper motor and ball-screw, with a total span of $0.2 \mathrm{~m}$ and resolution of $50 \mu \mathrm{m}$. For measuring interaction forces, each module has a beam-type biaxial load-cell (Sensor and Control Co., Ltd.), which can measure $160 \mathrm{~N}$ in $X$ and $80 \mathrm{~N}$ in $Y$ directions, with an accuracy of $0.08 \mathrm{~N}$ and $0.04 \mathrm{~N}$ respectively. By using one module per contact point, this modular platform enables the study of force distribution for a multi-contact loading condition. In this study, we use three modules, marked as 1 to 3 in Fig. 1 Modules 1 and 3 are arranged horizontally, co-axial with each other while module 2 is arranged vertically as shown in the figure. Each module consists of a mounting plate for affixing the attachments.

- Attachments: These are used for enforcing anchoring conditions. Based on the actuator used and constraints required, attachments are custom designed. The appropriate attachments are then affixed to the modules using the mounting plates, and then clamped to the actuator at the desired contact locations. In this work, we designed two types of attachments, one each for enforcing hinge and cantilever constraint. We fabricated them by 3D printing, followed by manual assembly.

- Frame: The frame provides structural support and allows for reconfigurability of the modules as it is fabricated using Tslotted aluminium profiles. In the current prototype, we can characterize actuators of different sizes, with a maximum span of $0.4 \mathrm{~m} \times 0.2 \mathrm{~m} \times 0.3 \mathrm{~m}$ in the XYZ space.

The position control of the modules is carried out openloop, using TB660 stepper motor drivers. Load-cell outputs are amplified using a 24-bit ADC, Hx711. The amplified and digitized sensor readings are processed by reading a 6-sample set, ignoring the highest and lowest reading, and then using a moving average filter for the remaining 4 samples. The loadcell data is read at an average of $7.75 \mathrm{~Hz}$, limited due to the use of HX711 amplifiers. Here, as we characterize in quasistatic loading conditions, a high sampling rate is not required. The stepper motor consumes current up to $2 \mathrm{~A}$, which can potentially induce electromagnetic noise in the load-cell signal. On investigation, however, we did not observe any such effects in our setup.

Prior to testing, the three load-cells were calibrated. Standard brass weights in increments of 50 grams were placed on each load-cell and their output was recorded and averaged for 10 s to get the six calibration factors ( $\mathrm{X}$ and $Y$ directions for each load-cell). Before the start of each test, the initial reading from each load-cell was noted. All readings during the test were measured with respect to this initial value, thus, corresponding to the differential force.

\section{B. Pulling force}

We clamped the two ends of the actuator with the hinged attachments, one each on module 1 and 3 as shown in Fig. 2A.
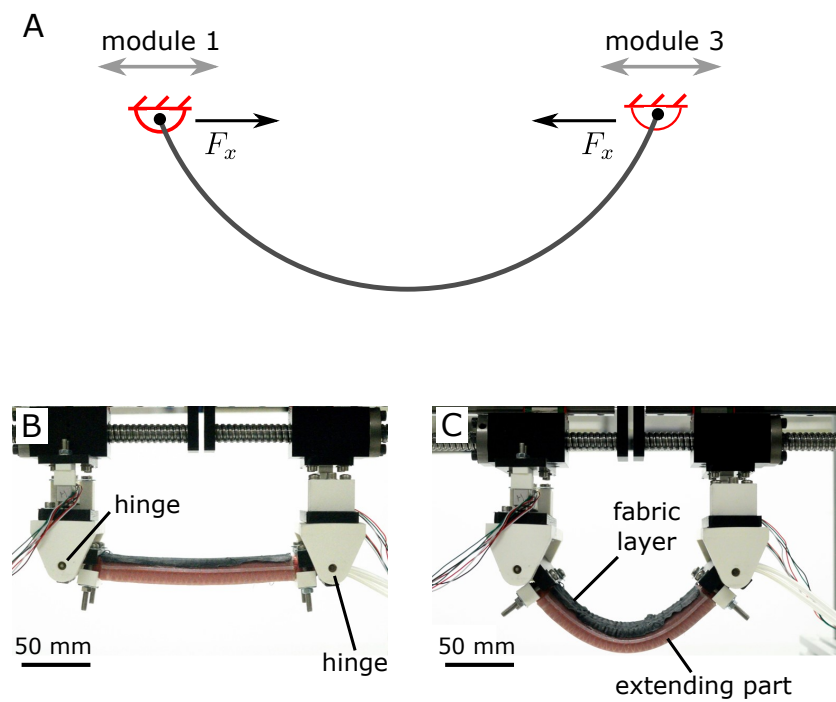

Fig. 2. Pulling force characterization of the soft bending actuator. (A) Anchoring conditions and measured forces: we used hinged attachments to anchor the two ends of the actuator on modules 1 and 3. $F_{x}$ is the mean horizontal force measured from the two modules. We took $F_{x}$ as positive when the forces applied by the actuator on the modules were in the direction of the arrows. (B) The actuator held flat between the modules, and (C) The actuator in bent configuration. When pressurized, actuator extension is prevented on one side by a fabric layer, thereby generating a bending motion.

1) Displacement boundary conditions: Actuator displacement in this condition depends only on the distance between the hinges due to free rotation at the ends. Therefore, we enforce linear displacement on the hinges to move the actuator from flat (Fig. 2B) to bent shape (Fig. 2C) and then back to initial position. We took the linear displacement as $0 \mathrm{~mm}$ when the hinges were furthest from each other. The maximum enforced linear displacement was $80 \mathrm{~mm}$ when the hinges were closest from each other.

2) Control and data measurement: We controlled modules 1 and 3 in opposite directions to enforce linear displacement to the tips of the actuator. For quasi-static conditions, the speed of the modules was maintained at $1.5 \mathrm{~mm} / \mathrm{s}$. We characterized the actuator pulling force for nine pressures from $0 \mathrm{kPa}$ to $200 \mathrm{kPa}$, in steps of $25 \mathrm{kPa}$, repeated thrice for each. The pressure was generated using an off-board compressor and controlled using a digital pressure regulator (SMC). The measured parameters were the horizontal and vertical forces from modules 1 and 3, pressure, and positions of the modules. While this includes four measurements from the load-cells, we expect the horizontal forces to be equal and opposite, while 
both vertical forces will be zero. We take this force, $F_{x}$, as the average horizontal force from modules 1 and 3, with the arrows in Fig. 2A depicting sign conventions.

\section{Tip loading}

We clamped one end of the actuator on module 1 with a cantilever attachment, and the other end with a hinge attachment on the module 2, as shown in Fig. 44.

1) Coordinate system: We define a new coordinate system to express the planar space around the actuator tip, as shown in Fig. 3 .

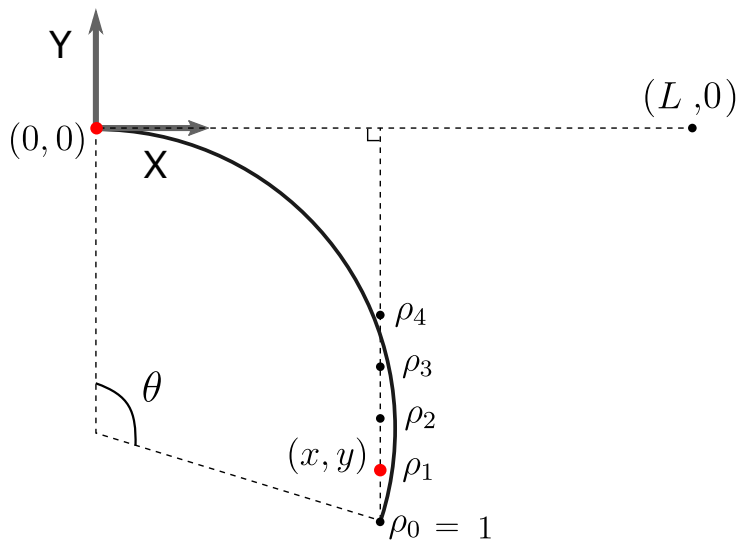

Fig. 3. Coordinate system for tip loading. $L$ is the arc length equal to actuator length, and $\theta$ is the arc curvature. $\rho$ scales the $Y$ coordinate of the arc endpoint. Using $\theta$ and $\rho$, we define the new coordinate system to express the planar space around the actuator tip. The two red points correspond to the locations of the actuator tips. One tip is at the origin, while the other is at $(x, y)$, which is given by Eq. 1

Consider an arc with one endpoint at the origin, curvature $\theta$ and arc length $L$ equal to length of the actuator. The second arc endpoint fixes the $X$-coordinate for a given value of $\theta$. We then define $\rho$, which determines the $Y$-coordinate, as shown in Fig. 3. Using this, we get a direct mapping from the $\theta-\rho$ space to the $X-Y$ space as follows:

$$
x=\frac{L \sin \theta}{\theta}, \quad y=\frac{L(\cos \theta-1) \rho}{\theta}
$$

Using $\theta$ and $\rho$, we define a region, shaded in Fig. $4 \mathrm{~B}$, around the tip of the actuator, given by the fixed intervals: $\theta=[0, \pi / 2]$ and $\rho=[0.6,1]$.

2) Displacement boundary conditions: We define a grid of points, using ten values of $\theta: 0, \pi / 18,2 \pi / 18, \ldots \pi / 2$; and five values of $\rho: 0.6,0.7,0.8,0.9,1$. These are highlighted in red in Fig. $4 \mathrm{~B}$. We then plan a trajectory to move and hold the hinged end of the actuator at each of these grid points sequentially. Starting from $\theta=0$ and $\rho=0.6$, we increase $\rho$ in steps of 0.1 , with a time interval of 10 s. Once $\rho$ reaches $1, \theta$ is incremented by $\pi / 20$, and $\rho$ is reset to 0.6 . The entire trajectory consists of increasing $\theta$ from 0 to $\pi / 2$ and then decreasing it back to 0 . With a total of 20 steps for $\theta$, each having 5 steps of $\rho$, a total of 100 steps are taken. While enforcing these displacement boundary conditions, the $\theta$ - $\rho$ coordinate system does not represent actuator shape, rather it corresponds to the location of the actuator tip.
A

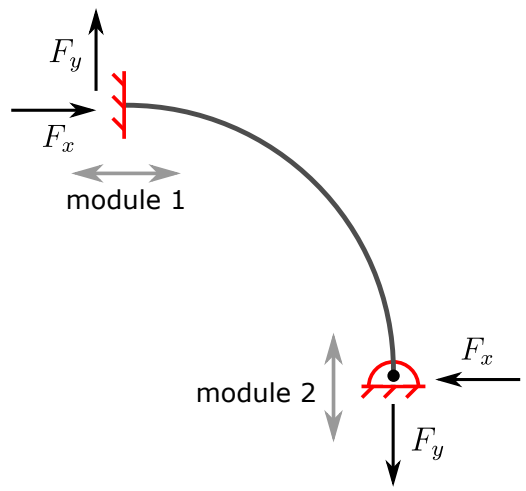

B
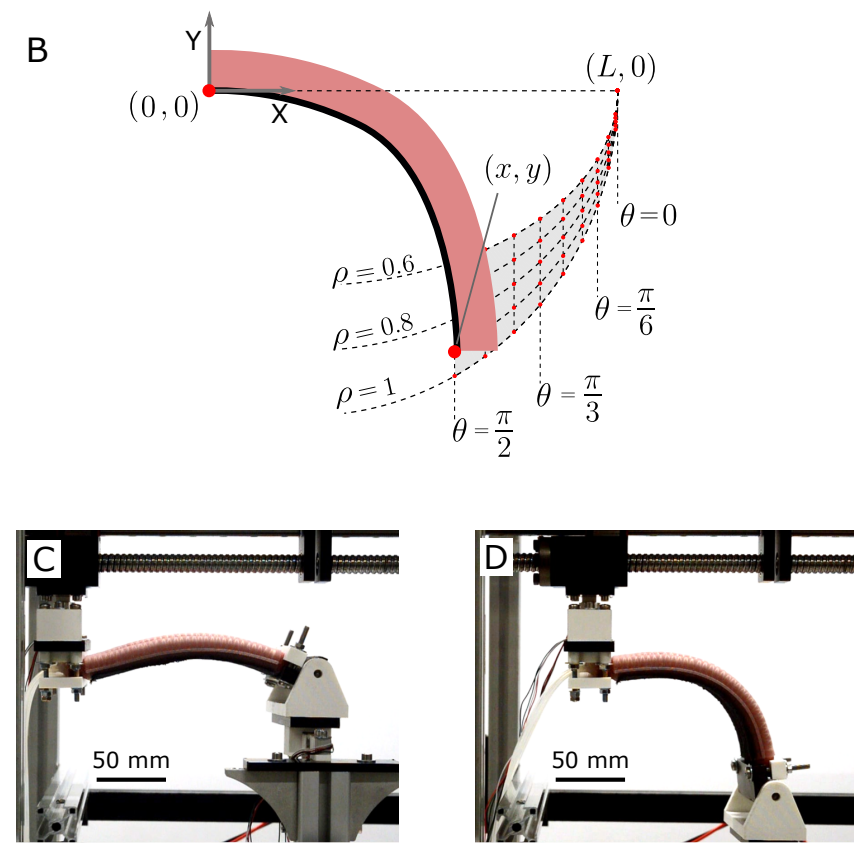

Fig. 4. Tip loading characterization of the actuator. (A) Anchoring conditions and measured forces: we constrained one end of the actuator in a cantilever attachment, and the other in a hinge attachment. $F_{x}$ and $F_{y}$ are the mean horizontal and vertical forces measured at modules 1 and 2. We took them as positive when the forces applied by the actuator were in the direction of the arrows, (B) Displacement boundary conditions: we moved the endpoint of the actuator to each grid point, marked red in the shaded region. The $X-Y$ coordinates of these grid points are calculated using Eq. 1 for ten values of $\theta: 0, \pi / 18,2 \pi / 18, \ldots \pi / 2$; and five values of $\rho: 0.6,0.7,0.8,0.9,1$, (C) The actuator held flat between the modules, represented by $\theta=0$, and (D) The actuator in bent configuration, represented by $\theta>0$.

3) Control and data measurement: To enforce the actuator to all the grid points defined earlier, module 1 followed the trajectory of $x$ and module 2 followed a trajectory of $y$. While doing so, we measured the interaction forces at the two modules. This was carried out for nine inflation pressures from $0 \mathrm{kPa}$ to $200 \mathrm{kPa}$ in steps of $25 \mathrm{kPa}$, and repeated thrice for each inflation pressure, to give a total of 27 runs, and a total time of 27,000 s ( 7.5 hours).

Here again, we expect both horizontal and vertical forces to be equal and opposite on the two modules. We took the mean horizontal and vertical forces acting on the modules, and denoted them as $F_{x}$ and $F_{y}$ respectively, as shown in Fig. $4 \mathrm{~A}$ with the arrows depicting sign conventions. 


\section{Symmetric three-point bending}

We clamped the two ends of the actuator with hinge attachments on modules 1 and 3 , and the middle section on module 2 with a cantilever attachment as shown in Fig. 6A.

1) Coordinate system: We define a new coordinate system to express the space around the actuator tip, as shown in Fig. 5

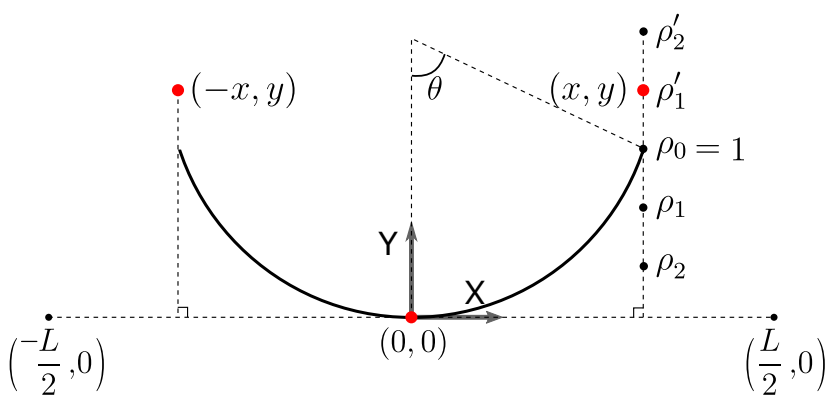

Fig. 5. Coordinate system for three-point bending. $L$ is the arc length equal to actuator length, and $\theta$ is the arc semi-curvature. $\rho$ scales the $Y$ coordinate of the arc endpoints. Using $\theta$ and $\rho$, we define the new coordinate system to express the planar space around the actuator tips. The red points correspond to the location of the three contact locations ends of the actuator. The central point is at the origin, while the two tips are at $(x, y)$ and $(-x, y)$, given by Eq. 2

Consider an arc with semi-curvature $\theta$, saddle point at the origin, and arc length $L$ equal to the length of the actuator. The arc endpoints fix the $X$-coordinates, $\pm x$, for a given value of $\theta$. We then define $\rho$, which determines the $Y$-coordinate, as shown in Fig. 5. Using this, we get a direct mapping from the $\theta-\rho$ space to the $X-Y$ space as follows:

$$
x=\frac{L \sin \theta}{2 \theta}, \quad y=\frac{L(1-\cos \theta) \rho}{2 \theta}
$$

Using $\theta$ and $\rho$, we define a region, shaded in Fig. 6B, around the tips of the actuator, given by the fixed intervals: $\theta=[0, \pi / 2]$ and $\rho=[0.8,1.2]$.

2) Displacement boundary conditions: We created a grid of points using ten values of $\theta: 0, \pi / 18,2 \pi / 18, \ldots \pi / 2$ and five values of $\rho: 0.8,0.9,1,1.1,1.2$. These grid points are highlighted in red in Fig. 6 6B. The ends of the actuator were symmetrically moved to each of these grid points sequentially, starting from $\theta=0, \rho=0.8$ to $\theta=\pi / 2, \rho=1.2$, and back. While enforcing these displacement boundary conditions, the $\theta-\rho$ coordinate system does not represent actuator shape, rather it corresponds to the location of the actuator tip.

3) Control and data measurement: To enforce the ends of the actuator to the grid points, modules 1 and 3 followed the trajectory of $-x$ and $x$ respectively, and module 2 followed a trajectory of $-y$. The actuator was held in each point in the grid and the interaction forces at the three locations were measured. This was carried out for nine inflation pressures from $0 \mathrm{kPa}$ to $200 \mathrm{kPa}$ in steps of $25 \mathrm{kPa}$, and repeated thrice for each inflation pressure, to give a total of 27 runs, and a total time of $27,000 \mathrm{~s}$ (7.5 hours).

In ideal conditions with perfect symmetry, we expect the horizontal force on module 2 to be zero. In practice, however, a residual force was observed as the actuator buckled to one
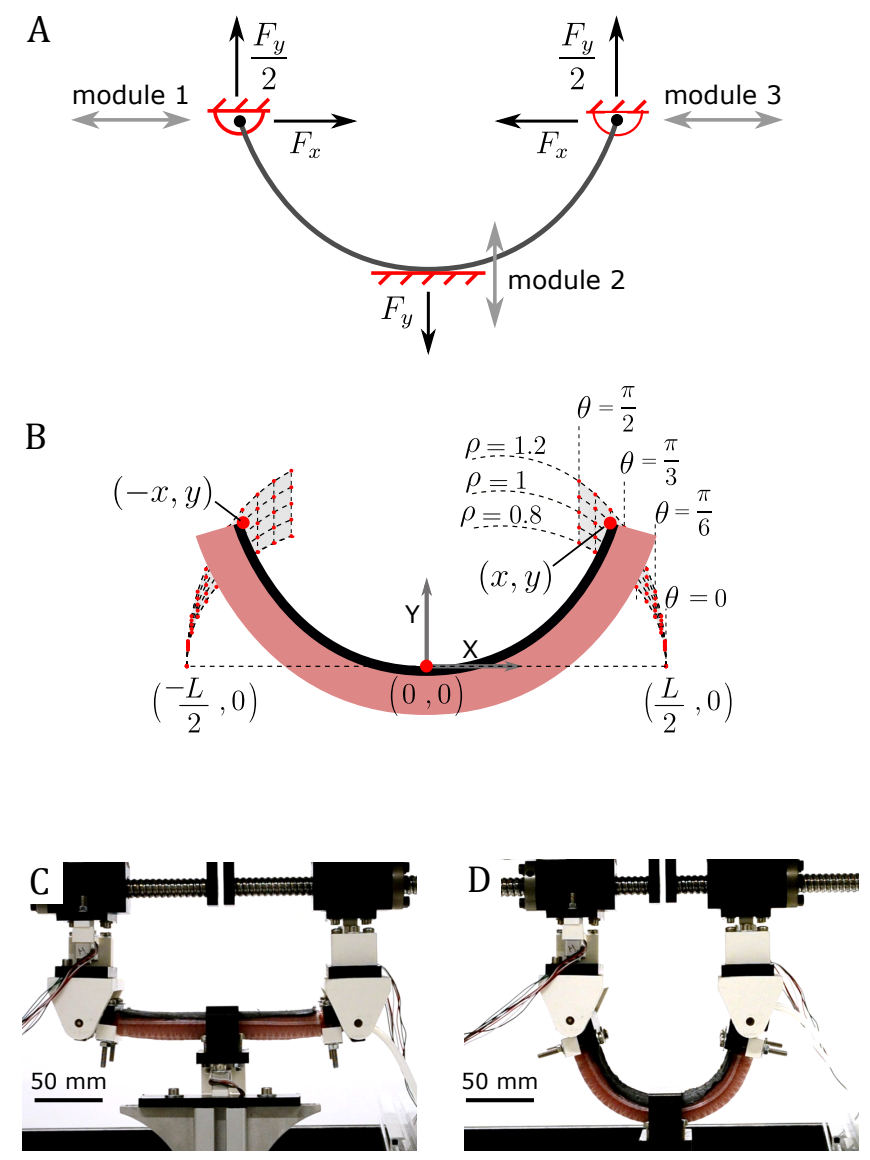

Fig. 6. Three-point bending characterization of the actuator. (A) Anchoring conditions and measured forces: we constrained the two ends of the actuator in hinge attachments and the middle section in a cantilever attachment. $F_{x}$ and $F_{y}$ are the mean horizontal and vertical forces from the modules. We took them as positive when the forces applied by the actuator were in the direction of the arrows. (B) Displacement boundary conditions: we symmetrically moved the two endpoints of the actuator to the grid points, marked red in the shaded region. The $X-Y$ coordinates of these grid points are calculated using Eq. 2 for ten values of $\theta: 0, \pi / 18,2 \pi / 18, \ldots \pi / 2$; and five values of $\rho: 0.8,0.9,1,1.1,1.2$, (C) The actuator held flat between the modules, represented by $\theta=0$, and (D) The actuator in bent configuration, represented by $\theta>0$.

of the sides. However, as it was almost an order of magnitude lower than the other forces, we only consider the vertical force component at module 2. For modules 1 and 3, we expect the horizontal forces to be equal and opposite due to symmetry. Furthermore, the vertical forces on modules 1 and 3 will be equal, and their sum will be equal to the vertical force on module 2. Thus, there are again two independent forces. We took $F_{x}$ as the mean horizontal force from modules 1 and 3, and $F_{y}$ as the mean vertical force from modules 1,2 and 3, as shown in Fig. 6A, with the arrows depicting sign conventions.

\section{RESUlTS AND DISCUSSION}

Here, we analyze how the measured interaction forces were affected by the anchoring conditions, displacement boundary conditions and actuation power.

\section{A. Pulling force}

Fig. 7 shows the actuator pulling force at four pressures. The pulling force increases with pressure and decreases with displacement. We also observe that the force vs. displacement 


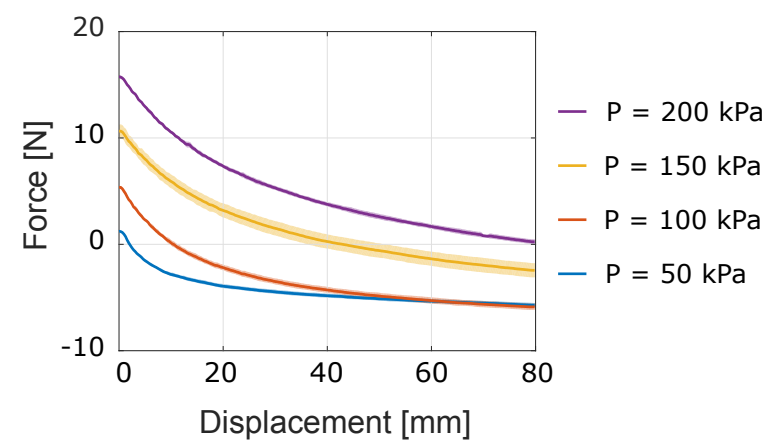

Fig. 7. Mean pulling force, $F_{x}$, of the actuator vs. displacement, $x$. The pulling force increases with pressure and decreases with $x$. The shaded region corresponds to one standard deviation of the measured data.

behaviour of the actuator shows a similar trend for the different pressures, with changing $X$-intercepts. These results are consistent with those from conventional studies characterizing SPA pulling force [9], [33], [36].

\section{B. Tip loading}

Fig. 8 shows the evolution of the tip horizontal force, $F_{x}$, at varying pressures, $\theta$ and $\rho$. We observe that for a given value of $\rho, F_{x}$ shows a similar trend with $\theta$ for different pressures. However, this trend changes significantly when $\rho$ is varied. Based on how we defined the $\theta-\rho$ coordinate system, we expected $F_{x}$ to always reduce with increasing $\theta$. However, from Fig. 8 we see that the opposite trend is observed for some values of $\theta, \rho$ and pressure. Our experimental protocol with planar displacement boundary conditions helps to capture this behaviour, which is otherwise not observable with conventional methods.

We used linear regression to fit a second-order model to the measured data as:

$$
F_{x, y}=f_{x, y}(P, x, y, \theta, \rho)
$$

where $f_{x, y}$ are second order models, $F_{x, y}$ are the measured forces, $P$ is the internal air pressure, $x$ and $y$ are the $X$ and $Y$ coordinates of the tip, and $\theta$ and $\rho$ represent the tip location using the coordinate system in Fig. 3 .

Table [1 shows the root mean square (RMS) errors between the fitted model and measured values. Additionally, the error
TABLE I

RMS ERRORS BETWEEN MEASURED VALUES AND THE REGRESSED MODEL FOR INTERACTION FORCES DURING TIP LOADING

\begin{tabular}{|c|c|c|}
\hline Force & Peak Force & RMS error \\
\hline$F_{x}$ & $12.63 \mathrm{~N}$ & $0.57 \mathrm{~N}$ \\
$F_{y}$ & $5.78 \mathrm{~N}$ & $0.58 \mathrm{~N}$ \\
\hline
\end{tabular}

bars in Fig. 8 also signify the RMS errors between the model and measured values. Despite rigorous testing of over 7 hours, the model is able to capture the soft actuator mechanical behaviour well, thus highlighting the repeatability of our experimental protocol.

\section{Three-point bending}

Fig. 9 shows the evolution of the vertical force, $F_{y}$, with varying pressures, $\theta$ and $\rho$. Similar to tip loading, the behaviour of force vs. $\theta$, shows a similar trend for a given $\rho$, at different pressures. With varying $\rho$, however, we observe markedly different behaviour. Additionally, we see that the force magnitude in three-point bending is in general, higher than both tip loading and pulling. This is because in the other two cases, the actuator is constrained only at the ends, and its remaining body is free to bend in the unconstrained sections. In case of three-point bending, an additional constraint acts at the central portion of the actuator, preventing its motion, thereby leading to higher interaction forces.

TABLE II

RMS ERRORS BETWEEN MEASURED VALUES AND THE REGRESSED MODEL FOR INTERACTION FORCES DURING THREE-POINT BENDING

\begin{tabular}{|c|c|c|}
\hline Force & Peak Force & RMS error \\
\hline$F_{x}$ & $40.15 \mathrm{~N}$ & $1.10 \mathrm{~N}$ \\
$F_{y}$ & $56.27 \mathrm{~N}$ & $1.48 \mathrm{~N}$ \\
\hline
\end{tabular}

Similar to tip loading, we used regression to fit a second order model to the measured data. The RMS errors are given in Table II] While the magnitudes of RMS errors are higher than those during tip loading, the relative errors are lower. Additionally, the error bars in Fig. 9 also signify the RMS errors between the model and measured values. As can be seen from the table and figure, the regressed model is able to capture the actuator mechanical behaviour closely.

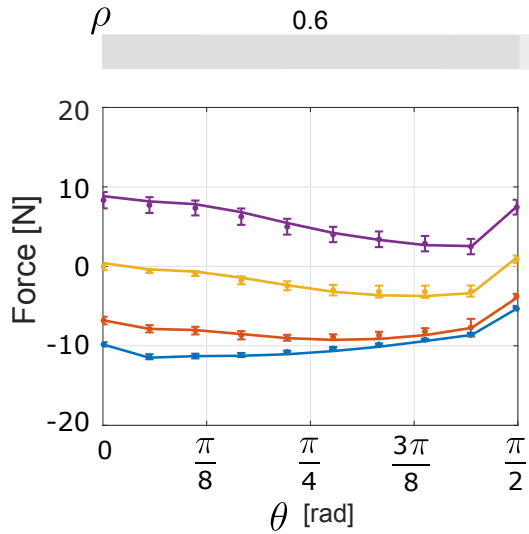

0.8
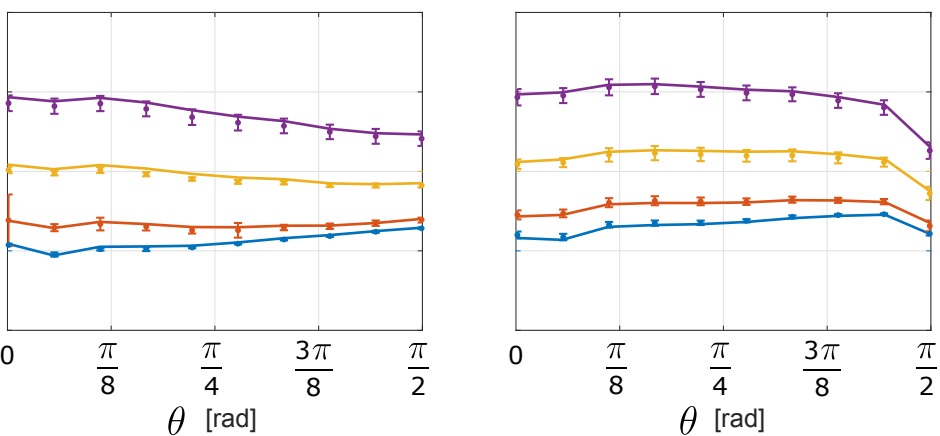

I $\mathrm{P}=200 \mathrm{kPa}$

I $\mathrm{P}=150 \mathrm{kPa}$

I $\mathrm{P}=100 \mathrm{kPa}$

I $\mathrm{P}=50 \mathrm{kPa}$

Fig. 8. Mean horizontal force, $F_{x}$, of the actuator during tip loading, measured at varying pressures, $\theta$ and $\rho$. The data markers correspond to measured values and the lines correspond to the fitted model. The error bars signify the RMS errors between the model and measured data. 


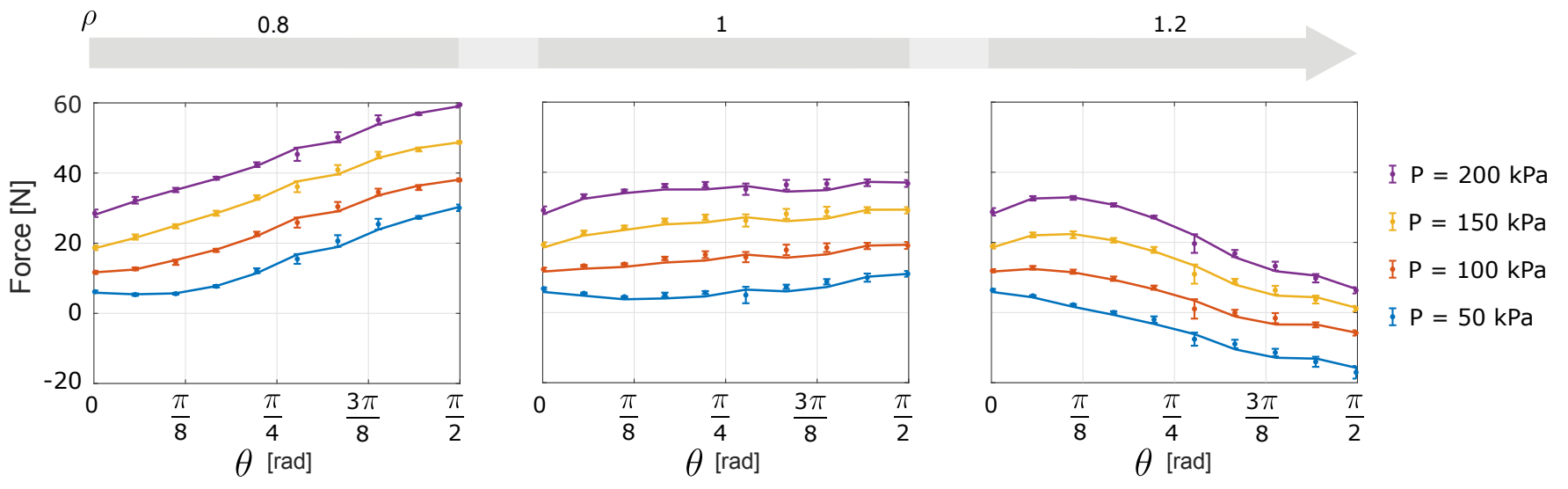

Fig. 9. Mean vertical force, $F_{y}$, of the actuator during three-point bending measured at varying pressures, $\theta$ and $\rho$. The data markers correspond to measured values, and the lines correspond to the fitted model. The error bars signify the RMS errors between the model and measured data.

A

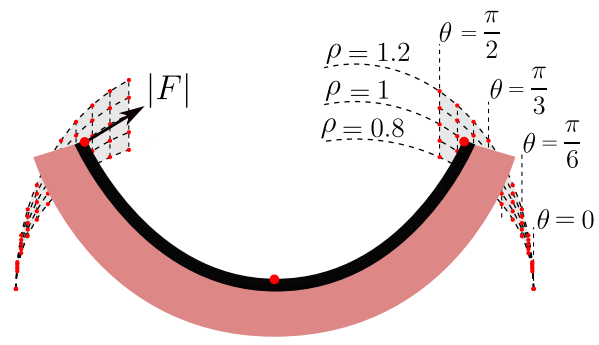

B

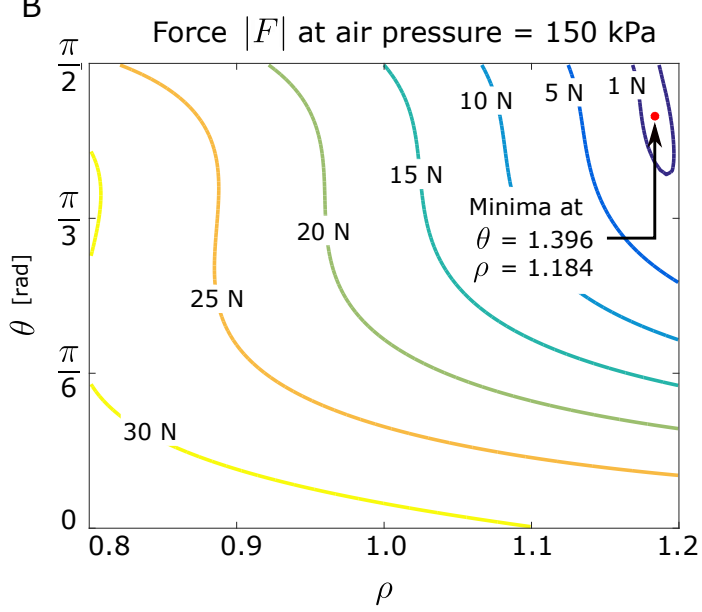

Fig. 10. Predicted resultant force, $|F|$, at the left tip of the actuator in threepoint bending; at an air pressure of $150 \mathrm{kPa}$. (A) Figure depicting planar displacement showing a grid of $\theta$ and $\rho$, and the resultant tip force with magnitude $|F|$, and (B) Magnitude of resultant force at varying $\theta$ and $\rho$. The point of minimum force $(|F|=0.244 \mathrm{~N})$ is marked in red.

Using the fitted model, we calculated the magnitude of the resultant force vector $\left(F_{x}, F_{y} / 2\right)$ acting on the tip of the actuator, for a range of $\theta$ and $\rho$. This is shown in Fig. 10 for an actuation pressure of $150 \mathrm{kPa}$. This contour plot represents the stiffness of the actuator in the planar space. Furthermore, the minima, marked in red, corresponds to the neutral position of the actuator when pressurized to $150 \mathrm{kPa}$, as it has the minimum resultant tip force $(|F|=0.244 \mathrm{~N})$. Comparing with the measured data, the displacement with minimum resultant force corresponds to $\theta=1.396$ and $\rho=1.2$, which is close to that predicted by the fitted model. As the magnitude of the force is almost zero, these values of $\theta$ and $\rho$ represent the actuator displacement in free or unconstrained conditions at an inflation pressure of $150 \mathrm{kPa}$.

\section{CONClusion}

In this paper, we presented a novel experimental protocol for characterizing soft actuator interaction forces by actively controlling displacement boundary conditions, anchoring conditions and actuation power. To implement such a multiDoF protocol, we designed a novel modular robotic test platform, which can extensively characterize soft actuators in a diverse set of loading conditions. Using this platform, we tested a bending soft actuator in three loading conditions and observed distinct mechanical performance in the three cases. This systematic protocol helps to closely recreate true loading conditions, which leads to accurate and repeatable prediction of soft actuator performance. The methodology presented in this paper is a step towards better understanding the impact of loading conditions on actuator mechanical behaviour. This can contribute towards optimization of soft actuator design and control, thereby enhancing their scope of application.

The presented experimental protocol for characterization can be readily applied for other core robotic components such as soft sensors and electronics, as well as traditional rigid devices. While we limited our study to quasi-static testing of a bending actuator in a single plane, future developments will investigate dynamic force characterization of different actuator types in the 3-D space. Additionally, we will incorporate more modules for studying force distribution at multiple contact points.

\section{REFERENCES}

[1] Daniela Rus and Michael T Tolley. Design, fabrication and control of soft robots. Nature, 521(7553):467-475, 2015.

[2] Gunjan Agarwal, Nicolas Besuchet, Basile Audergon, and Jamie Paik. Stretchable materials for robust soft actuators towards assistive wearable devices. Scientific reports, 6:34224, 2016.

[3] Ramses V Martinez, Ana C Glavan, Christoph Keplinger, Alexis I Oyetibo, and George M Whitesides. Soft actuators and robots that are resistant to mechanical damage. Advanced Functional Materials, 24(20):3003-3010, 2014. 
[4] Yong-Lae Park, Bor-rong Chen, Néstor O Pérez-Arancibia, Diana Young, Leia Stirling, Robert J Wood, Eugene C Goldfield, and Radhika Nagpal. Design and control of a bio-inspired soft wearable robotic device for ankle-foot rehabilitation. Bioinspiration \& biomimetics, 9(1):016007, 2014.

[5] Yong-Lae Park, Jobim Santos, Kevin G Galloway, Eugene C Goldfield, and Robert J Wood. A soft wearable robotic device for active knee motions using flat pneumatic artificial muscles. In Robotics and Automation (ICRA), 2014 IEEE International Conference on, pages 4805-4810. IEEE, 2014.

[6] Harshal Arun Sonar and Jamie Paik. Soft pneumatic actuator skin with piezoelectric sensors for vibrotactile feedback. Frontiers in Robotics and AI, 2:38, 2016.

[7] Hong Kai Yap, Nazir Kamaldin, Jeong Hoon Lim, Fatima Nasrallah, James $\mathrm{CH}$ Goh, and Chen-Hua Yeow. A magnetic resonance compatible soft wearable robotic glove for hand rehabilitation and brain imaging. IEEE transactions on neural systems and rehabilitation engineering, 2016.

[8] Michael Wehner, Brendan Quinlivan, Patrick M Aubin, Ernesto Martinez-Villalpando, Michael Baumann, Leia Stirling, Kenneth Holt, Robert Wood, and Conor Walsh. A lightweight soft exosuit for gait assistance. In Robotics and Automation (ICRA), 2013 IEEE International Conference on, pages 3362-3369. IEEE, 2013.

[9] Hong Kai Yap, Benjamin WK Ang, Jeong Hoon Lim, James CH Goh, and Chen-Hua Yeow. A fabric-regulated soft robotic glove with user intent detection using emg and rfid for hand assistive application. In Robotics and Automation (ICRA), 2016 IEEE International Conference on, pages 3537-3542. IEEE, 2016.

[10] Victoria Oguntosin, William S Harwin, Sadao Kawamura, Slawomir J Nasuto, and Yoshikatsu Hayashi. Development of a wearable assistive soft robotic device for elbow rehabilitation. In Rehabilitation Robotics (ICORR), 2015 IEEE International Conference on, pages 747-752. IEEE, 2015.

[11] Michael T Tolley, Robert F Shepherd, Bobak Mosadegh, Kevin C Galloway, Michael Wehner, Michael Karpelson, Robert J Wood, and George M Whitesides. A resilient, untethered soft robot. Soft Robotics, 1(3):213-223, 2014

[12] Robert F Shepherd, Filip Ilievski, Wonjae Choi, Stephen A Morin, Adam A Stokes, Aaron D Mazzeo, Xin Chen, Michael Wang, and George M Whitesides. Multigait soft robot. Proceedings of the National Academy of Sciences, 108(51):20400-20403, 2011.

[13] Cagdas D Onal and Daniela Rus. Autonomous undulatory serpentine locomotion utilizing body dynamics of a fluidic soft robot. Bioinspiration \& biomimetics, 8(2):026003, 2013.

[14] Andrew D Marchese, Cagdas D Onal, and Daniela Rus. Autonomous soft robotic fish capable of escape maneuvers using fluidic elastomer actuators. Soft Robotics, 1(1):75-87, 2014.

[15] Ming Luo, Mahdi Agheli, and Cagdas D Onal. Theoretical modeling and experimental analysis of a pressure-operated soft robotic snake. Soft Robotics, 1(2):136-146, 2014.

[16] Jiawei Cao, Lei Qin, Jun Liu, Qinyuan Ren, Choon Chiang Foo, Hongqiang Wang, Heow Pueh Lee, and Jian Zhu. Untethered soft robot capable of stable locomotion using soft electrostatic actuators. Extreme Mechanics Letters, 21:9-16, 2018.

[17] Adam A Stokes, Robert F Shepherd, Stephen A Morin, Filip Ilievski, and George M Whitesides. A hybrid combining hard and soft robots. Soft Robotics, 1(1):70-74, 2014.

[18] Filip Ilievski, Aaron D Mazzeo, Robert F Shepherd, Xin Chen, and George M Whitesides. Soft robotics for chemists. Angewandte Chemie, 123(8):1930-1935, 2011.

[19] Mariangela Manti, Taimoor Hassan, Giovanni Passetti, Nicolò D'Elia, Cecilia Laschi, and Matteo Cianchetti. A bioinspired soft robotic gripper for adaptable and effective grasping. Soft Robotics, 2(3):107-116, 2015

[20] Valentin Falkenhahn, Alexander Hildebrandt, Rüdiger Neumann, and Oliver Sawodny. Model-based feedforward position control of constant curvature continuum robots using feedback linearization. In Robotics and Automation (ICRA), 2015 IEEE International Conference on, pages 762-767. IEEE, 2015.
[21] Andrew D Marchese and Daniela Rus. Design, kinematics, and control of a soft spatial fluidic elastomer manipulator. The International Journal of Robotics Research, 35(7):840-869, 2016.

[22] Deepak Trivedi, Amir Lotfi, and Christopher D Rahn. Geometrically exact models for soft robotic manipulators. IEEE Transactions on Robotics, 24(4):773-780, 2008

[23] Federico Renda, Michele Giorelli, Marcello Calisti, Matteo Cianchetti, and Cecilia Laschi. Dynamic model of a multibending soft robot arm driven by cables. IEEE Transactions on Robotics, 30(5):1109-1122, 2014.

[24] Panagiotis Polygerinos, Zheng Wang, Johannes TB Overvelde, Kevin C Galloway, Robert J Wood, Katia Bertoldi, and Conor J Walsh. Modeling of soft fiber-reinforced bending actuators. IEEE Transactions on Robotics, 31(3):778-789, 2015.

[25] Andrew D Marchese, Russ Tedrake, and Daniela Rus. Dynamics and trajectory optimization for a soft spatial fluidic elastomer manipulator. The International Journal of Robotics Research, 35(8):1000-1019, 2016.

[26] Cosimo Della Santina, Robert K Katzschmann, Antonio Bicchi, and Daniela Rus. Dynamic control of soft robots interacting with the environment. In IEEE International Conference on Soft Robotics 2018. IEEE, 2018

[27] Christian Duriez. Control of elastic soft robots based on real-time finite element method. In Robotics and Automation (ICRA), 2013 IEEE International Conference on, pages 3982-3987. IEEE, 2013.

[28] Francois Faure, Christian Duriez, Hervé Delingette, Jérémie Allard, Benjamin Gilles, Stéphanie Marchesseau, Hugo Talbot, Hadrien Courtecuisse, Guillaume Bousquet, Igor Peterlik, et al. Sofa: A multimodel framework for interactive physical simulation. In Soft Tissue Biomechanical Modeling for Computer Assisted Surgery, pages 283321. Springer, 2012.

[29] Philip Moseley, Juan Manuel Florez, Harshal Arun Sonar, Gunjan Agarwal, William Curtin, and Jamie Paik. Modeling, design, and development of soft pneumatic actuators with finite element method. Advanced Engineering Materials, 18(6):978-988, 2016

[30] Bobak Mosadegh, Panagiotis Polygerinos, Christoph Keplinger, Sophia Wennstedt, Robert F Shepherd, Unmukt Gupta, Jongmin Shim, Katia Bertoldi, Conor J Walsh, and George M Whitesides. Pneumatic networks for soft robotics that actuate rapidly. Advanced Functional Materials, 24(15):2163-2170, 2014

[31] Cagdas D Onal and Daniela Rus. A modular approach to soft robots. In Biomedical Robotics and Biomechatronics (BioRob), 2012 4th IEEE RAS \& EMBS International Conference on, pages 1038-1045. IEEE, 2012.

[32] Yoel Shapiro, Alon Wolf, and Kosa Gabor. Bi-bellows: Pneumatic bending actuator. Sensors and Actuators A: Physical, 167(2):484-494, 2011.

[33] Andrew D Marchese, Robert K Katzschmann, and Daniela Rus. A recipe for soft fluidic elastomer robots. Soft Robotics, 2(1):7-25, 2015.

[34] Zheng Wang, Panagiotis Polygerinos, Johannes TB Overvelde, Kevin C Galloway, Katia Bertoldi, and Conor J Walsh. Interaction forces of soft fiber reinforced bending actuators. IEEE/ASME Transactions on Mechatronics, 22(2):717-727, 2017.

[35] Yong-Lae Park, Bor-rong Chen, Carmel Majidi, Robert J Wood, Radhika Nagpal, and Eugene Goldfield. Active modular elastomer sleeve for sof wearable assistance robots. In Intelligent Robots and Systems (IROS) 2012 IEEE/RSJ International Conference on, pages 1595-1602. IEEE, 2012.

[36] Yi Sun, Yun Seong Song, and Jamie Paik. Characterization of silicone rubber based soft pneumatic actuators. In 2013 IEEE/RSJ International Conference on Intelligent Robots and Systems, pages 4446-4453. Ieee, 2013.

[37] Guoying Gu, Jiang Zou, Ruike Zhao, Xuanhe Zhao, and Xiangyang Zhu. Soft wall-climbing robots. Science Robotics, 3(25):eaat2874, 2018.

[38] Huaxia Guo, Jinhua Zhang, Tao Wang, Yuanjie Li, Jun Hong, and Yue Li. Design and control of an inchworm-inspired soft robot with omegaarching locomotion. In 2017 IEEE International Conference on Robotics and Automation (ICRA), pages 4154-4159. IEEE, 2017.

[39] Matthew A Robertson, Hamed Sadeghi, Juan Manuel Florez, and Jamie Paik. Soft pneumatic actuator fascicles for high force and reliability. Soft Robotics, 4(1):23-32, 2017. 